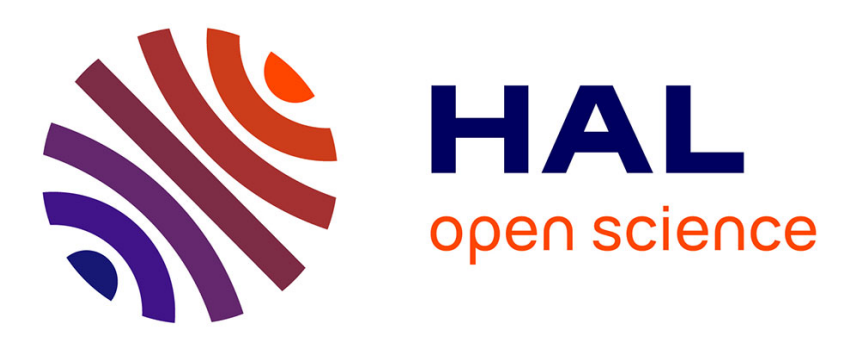

\title{
Laser direct writing of arbitrary complex polymer microstructures by nitroxide-mediated photopolymerization
}

Siham Telitel, Jason C Morris, Yohann Guillaneuf, Jean-Louis Clément, Fabrice Morlet-Savary, Arnaud Spangenberg, Jean-Pierre Malval, Jacques Lalevée, Didier Gigmes, Olivier Soppera

\section{To cite this version:}

Siham Telitel, Jason C Morris, Yohann Guillaneuf, Jean-Louis Clément, Fabrice Morlet-Savary, et al.. Laser direct writing of arbitrary complex polymer microstructures by nitroxide-mediated photopolymerization. ACS Applied Materials \& Interfaces, 2020, 12 (27), pp.30779-30786. 10.1021/acsami.0c06339 . hal-02997174

\section{HAL Id: hal-02997174 \\ https://hal.science/hal-02997174}

Submitted on 9 Nov 2020

HAL is a multi-disciplinary open access archive for the deposit and dissemination of scientific research documents, whether they are published or not. The documents may come from teaching and research institutions in France or abroad, or from public or private research centers.
L'archive ouverte pluridisciplinaire HAL, est destinée au dépôt et à la diffusion de documents scientifiques de niveau recherche, publiés ou non, émanant des établissements d'enseignement et de recherche français ou étrangers, des laboratoires publics ou privés. 


\title{
Laser direct writing of arbitrary complex polymer microstructures by nitroxide-mediated
}

\section{photopolymerization}

\author{
Siham Telitel ${ }^{1,2}$, Jason C. Morris ${ }^{3}$,Yohann Guillaneuf ${ }^{3}$, Jean-Louis Clément ${ }^{3}$, \\ Fabrice Morlet-Savary $^{1,2}$, Arnaud Spangenberg ${ }^{1,2}$, Jean-Pierre Malval ${ }^{1,2}$, Jacques Lalevée ${ }^{1,2}$, \\ Didier Gigmes ${ }^{3}$, Olivier Soppera ${ }^{*^{1,2}}$ \\ ${ }^{1}$ Université de Haute-Alsace, CNRS, IS2M UMR 7361, F-68100 Mulhouse, France \\ ${ }^{2}$ Université de Strasbourg, France \\ ${ }^{3}$ Aix Marseille Univ, CNRS, ICR UMR 7273, Marseille, F-13397, France \\ e-mail: olivier.soppera@uha.fr
}

\begin{abstract}
In this paper, we demonstrate the possibility of generating arbitrary polymer microstructures covalently linked to a first polymer layer by laser direct writing. At the molecular scale, the process relies on nitroxide-mediated photopolymerization (NMP2) triggered by a light-sensitive alkoxyamine. In addition to the proof-of-concept and examples of achievable structures, including multichemistry patterns and 3D structures, this paper aims at investigating the physicochemical phenomena involved under such conditions. In particular, the parameters influencing the repolymerization process are considered, and special attention is paid to the study of the impact of oxygen on the spatial control of the polymerization. Such work opens many possibilities towards the fabrication of on-demand highresolution (multi)functional polymer micro- and nanostructures.
\end{abstract}

\section{Keywords}

Laser direct writing, reversible deactivation radical polymerization, nitroxide-mediated photopolymerization, alkoxyamine, polymer micropatterning, oxygen, repolymerization, surface functionalization.

\section{Introduction}


Laser direct writing (LDW), also called maskless lithography, has attracted renewed interest in recent years due to the progress in high-resolution writing or applications to 3D printing. [1] Laser irradiation has specific properties, such as a high power density, coherence and a low divergence, such that the light pattern can be controlled with high accuracy at the submicron level over a wide range of wavelengths, from deep-UV to near-IR wavelengths, on a wide range of materials, including polymers. $[2,3,4,5,6]$ In laser direct writing, arbitrary patterns can be generated on demand without the need for special design of specific masks. Moreover, the possibility of delivering a high quantity of energy in a limited space and time makes it possible to initiate specific photoinduced physical, physicochemical or chemical transformations triggered by one or multiphoton absorption that can hardly be achieved by UV lamps. For these reasons, lasers were recently particularly used in practical applications for patterning polymers, such as ablation, melting, marking, welding, surface modification or $3 \mathrm{D}$ printing. $[7,8]$

In polymer additive manufacturing, laser direct writing has mostly been applied to free radical and epoxy resists. To extend the range of materials used in this context, several routes have been proposed to post-functionalize the microstructures. The first studies were based on post-functionalization using the unreacted polymerizing groups on the polymer surface. Either acrylate or epoxide groups could be reacted with nucleophiles $[9,10,11,12]$, allowing the grafting of various coating layers. However, the low amount of available reacting groups and the non-spatially resolved functionalization are the main limitations of these techniques. Yang et al. [13] went a step further by introducing a vinyl-terminated ATRP initiator into a UV curable resin to allow surface-initiated atom transfer radical polymerization (SI-ATRP). PEG- and perfluoro-based methacrylates could be grown from the surface of the object. Grubbs and Greer [14] also proposed to pre-functionalize a part of the resin by performing thiolMichael addition on one function of the multifunctional acrylate. In this case, the degree of functionalization was higher, but no example of localized functionalization was shown. Spatial control of repolymerization could also be shown for the photo-ATRP technique under excitation with a laser in the visible range $(532 \mathrm{~nm})$. [15]

More recently, methods based on the post-modification of surfaces or 3D structures by coating with substances bearing reactive moieties that can be addressed by efficient ligation methods have been 
proposed by the groups of Wegener and Barner-Kowollik. [16,17] Various surface functionalization methods in combination with effective light-triggered transformations enable the formation of welldefined surface patterns with spatiotemporal control. The same teams also prepared a new resin combining a multifunctional acrylate with two methacrylate derivatives containing orthogonal photoreactive groups that could lead to a dual functionalization pattern. [18] Other recent works demonstrated the possibility of functionalizing gels by visible light photoredox catalysis using endlinked polymer gels embedded with trithiocarbonate iniferters. [19]

Among all these methods, those based on reversible deactivation radical polymerization (RDRP) have the important advantage to allow the reactivation of the polymer chains, as targeted in this paper. $[20,21,22,23]$ They include photoATRP and photoRAFT that have been successfully applied to modification of $2 \mathrm{D}$ surfaces and 3D printed pieces. $[24,25,26]$ They are compatible with UV lamp, laser and LED irradiation, with recent development of systems photosensitive from UV to the visible range.

In a previous study, we demonstrated the possibility of applying reversible deactivation radical polymerization (RDRP) (formerly known as controlled radical polymerization (CRP)) to graft polymer layers on polymer surfaces by light. Using light-sensitive alkoxyamines, it was possible to obtain good spatial control of the polymerized volume at the microscale by using a nitroxide-mediated photopolymerization (NMP2) process. Most of the previous works were carried out by using UV lamps and binary masks. [27] One of the drawbacks of this approach is that the generated pattern is a replica of the pattern written on the mask. Production of any other pattern requires the use of another mask. The proof of concept of using laser irradiation in the UV for NMP2 has been briefly reported, but no systematic study has been reported thus far. [28] By increasing the laser power, an increase in the line height was observed. Remarkably, the pattern height could be tuned from nm to microns with very good accuracy.

In this study, we show that we can extend the local reactivation by NMP2 by using laser direct writing. One of the advantages of this method is that the polymer structures are directly generated from the dormant species present at the surface. Arbitrary microstructures can thus be generated in a single step, unlike most of other processes proposed before. Special attention is then paid to the spatial 
control of the repolymerization reaction. It should be emphasized that the irradiation conditions are quite different for both configurations. The shift from mask lithography to laser direct writing is thus not trivial, which justifies a separate complete parametric study.

The irradiation parameters are given in Table 1.

Table 1. Comparison between experimental conditions for thermal and light-assisted nitroxidemediated polymerization in the case of lamp and laser irradiation.

\begin{tabular}{llll}
\hline & Thermal & UV Lamp & Laser $(\mathbf{0 . 2 3 5} \boldsymbol{\mu W})^{* *}$ \\
\hline Reaction Time & Few hours & $10 \mathrm{~s}$ & $10 \mathrm{~ms}$ \\
\hline Power $\left(\mathrm{mW} / \mathrm{cm}^{2}\right)$ & $/$ & 22 & 1200 \\
\hline Dose $\left(\mathrm{mJ} / \mathrm{cm}^{2}\right)$ & $/$ & 220 & 12 \\
\hline Medium & Solvent & Bulk monomer & Bulk monomer
\end{tabular}

* Typical values from ref $[27,28]$

** This work

In Table 1, we can note that the ratio of the irradiation times is 1000 , and that of the energies is 20 , which may lead to very different physicochemical conditions for the repolymerization reaction on the surface. The repolymerization reaction is expected to be dependent not only on the light dose (total amount of photons irradiating the surface) but also on the dynamic parameters driven by the light power density (proportional to the flux of photons). Moreover, unlike the UV lamp irradiation carried out in previous studies, the laser irradiation is performed at the microscale, which implies that the diffusion timescale of reactive species (photoinitiator, monomer, inhibitors) is different from that observed for macroscale irradiation.

The objective of this paper is thus to better investigate and understand the specific phenomena that occur during repolymerization triggered by laser irradiation. In particular, using laser direct writing, it is possible to reach high in-plane resolution, which extends the possibilities of this technique to highresolution (multi)functional polymer micro- and nanostructures. 


\section{Experimental}

Chemicals. All monomers were used as received. Ebecryl 605 (E605) was purchased from Allnex. It is composed of $75 \mathrm{wt} \%$ bisphenol A epoxy diacrylate and $25 \mathrm{wt} \%$ tripropylene glycol diacrylate. Trimethylolpropane triacrylate (TMPTA) and 2-hydroxyethyl acrylate (HEA) were purchased from Sigma-Aldrich. The synthesis of the alkoxyamine (AA) used in this work is described in [28]. The formulation typically contained $1 \mathrm{wt} \%$ AA in Ebecryl 605.

Sample preparation. The fabrication process is depicted in Figure 1. In the first step, a uniform film was prepared by photopolymerization. For this, a drop of the formulation was deposited on a glass slide and covered by a microscope cover slip before UV irradiation. UV curing was performed with a Hg-Xe UV lamp (Hamamatsu) equipped with a $365 \mathrm{~nm}$ bandpass filter at room temperature. Typical conditions were $50 \mathrm{~s}$ at $22 \mathrm{~mW} / \mathrm{cm}^{2}$.

The glass slide cover layer was then removed for repolymerization. A drop of a second monomer was deposited on the polymer surface. A microscope coverslip was placed on top of the monomer. The sample was then irradiated by the laser using the system described below. The laser was focused at the polymer/monomer interface to initiate the repolymerization. After irradiation, the sample was washed with isopropanol to remove the unreacted monomer at the surface of the sample. 


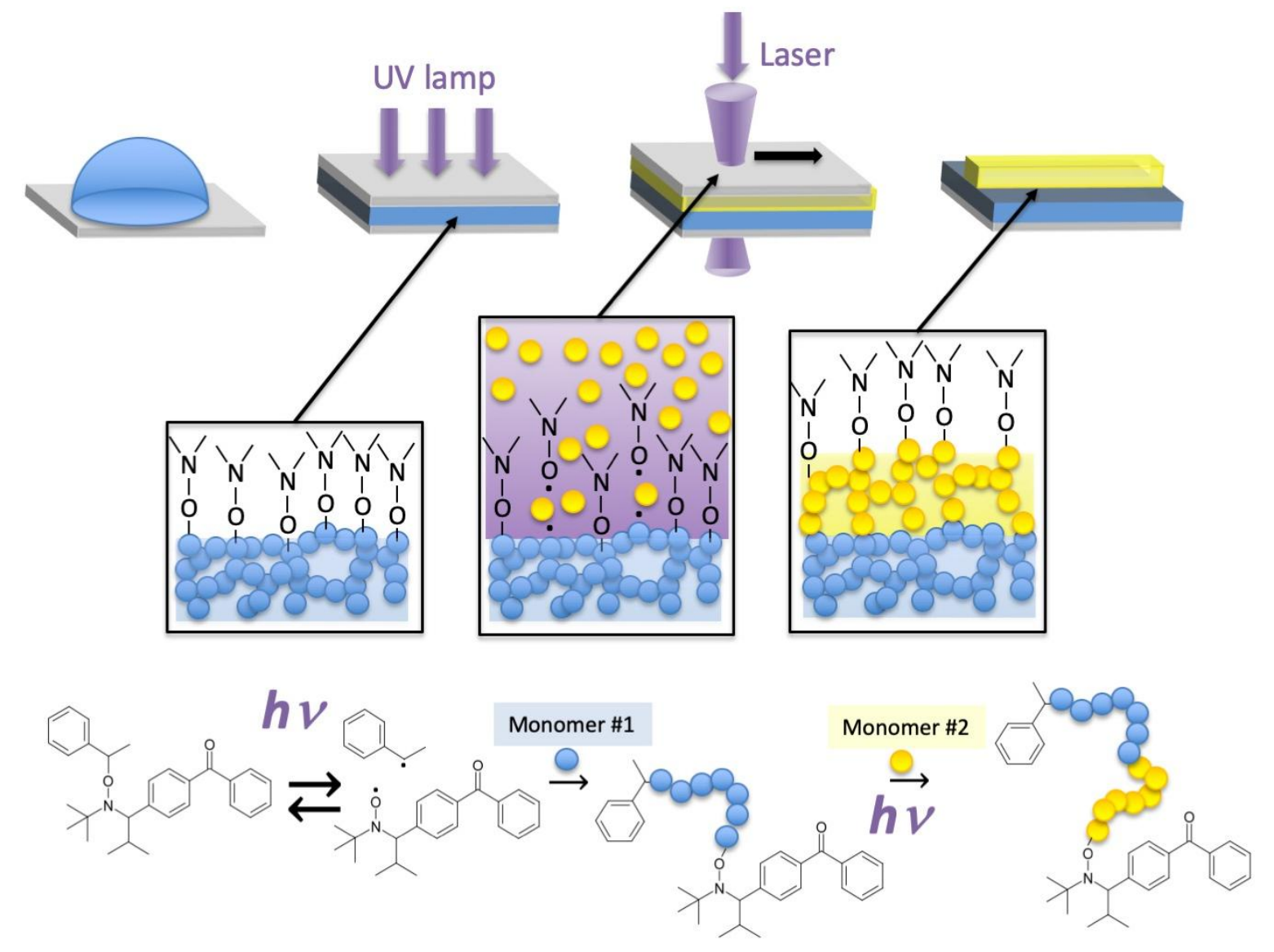

Figure 1: Schematic of the laser direct writing process by NMP2

The system for laser direct writing is based on a laser diode emitting at $375 \mathrm{~nm}$ (Cube from Coherent, $15 \mathrm{~mW}$ ). A spatial filter was used to circularize the beam and generate a Gaussian profile. The beam was then injected into a Zeiss Axio Observer DA microscope. Two types of microscope objectives were used to fabricate the microstructures: $\mathrm{x} 40(\mathrm{NA}=0.65$, air) and $\mathrm{x} 100(\mathrm{NA}=1.25$, oil). The sample was placed on a $(\mathrm{x}, \mathrm{y}, \mathrm{z})$ piezoelectric translation stage that could control the movement of the sample within a 300x300x300 $\mu \mathrm{m}^{3}$ volume. The translation stage was controlled by software provided by TeemPhotonics. The writing parameters were tuned by the software. The microstructures could be visualized during their fabrication by a camera installed on the microscope.

Sample characterization. The structures generated at the surface of the film were visualized by a Zeiss Axio Observer D1 optical microscope. Their topology was also characterized by a PicoPlus AFM in contact mode (force constant of $0.2 \mathrm{~N} / \mathrm{m}$ ). In particular, AFM was used to measure the height and width of laser-written microstructures. The height is measured from AFM profiles, between the 
first polymer surface and the top of the polymer structures. Nine measurements each time were made from which we calculated the average value and the standard deviation. The detection of very thin polymer structures (height $<10 \mathrm{~nm}$ ) was possible because of the very low roughness of the initial polymer film (estimated by AFM to 3-4 nm) that corresponds to the roughness of the glass coverslip used to polymerize the first polymer layer.

\section{Results and discussion}

The selectivity of $\mathrm{N}-\mathrm{O}$ vs. C-O photocleavage is a key parameter for efficient nitroxide radicals formation (only C-O cleavage must occur for NMP2 process). The selected AA was selected from [27] with a good selectivity for such a cleavage process leading to a potential use for surface patterning. The first proof of concept of laser writing based on these systems is provided by writing polymer patterns with TMPTA monomer on a first polymer film prepared with E605/AA. For repolymerization, the laser beam ( $375 \mathrm{~nm}$ ) was set to $3 \mu \mathrm{W}$. Microstructures were formed using the x100 magnification objective. The structures produced were grids with $20 \mu \mathrm{m}$ steps between each line. They were created by writing in a first step parallel lines in $\mathrm{x}$ direction and then another set of parallel line in y direction. Exposure times from 10 to $80 \mathrm{~ms}$ were used for each grid. These exposure times correspond to the exposure time needed to write the pixels that compose the line. The structures observed by optical microscopy and AFM are shown in Figure 2. 

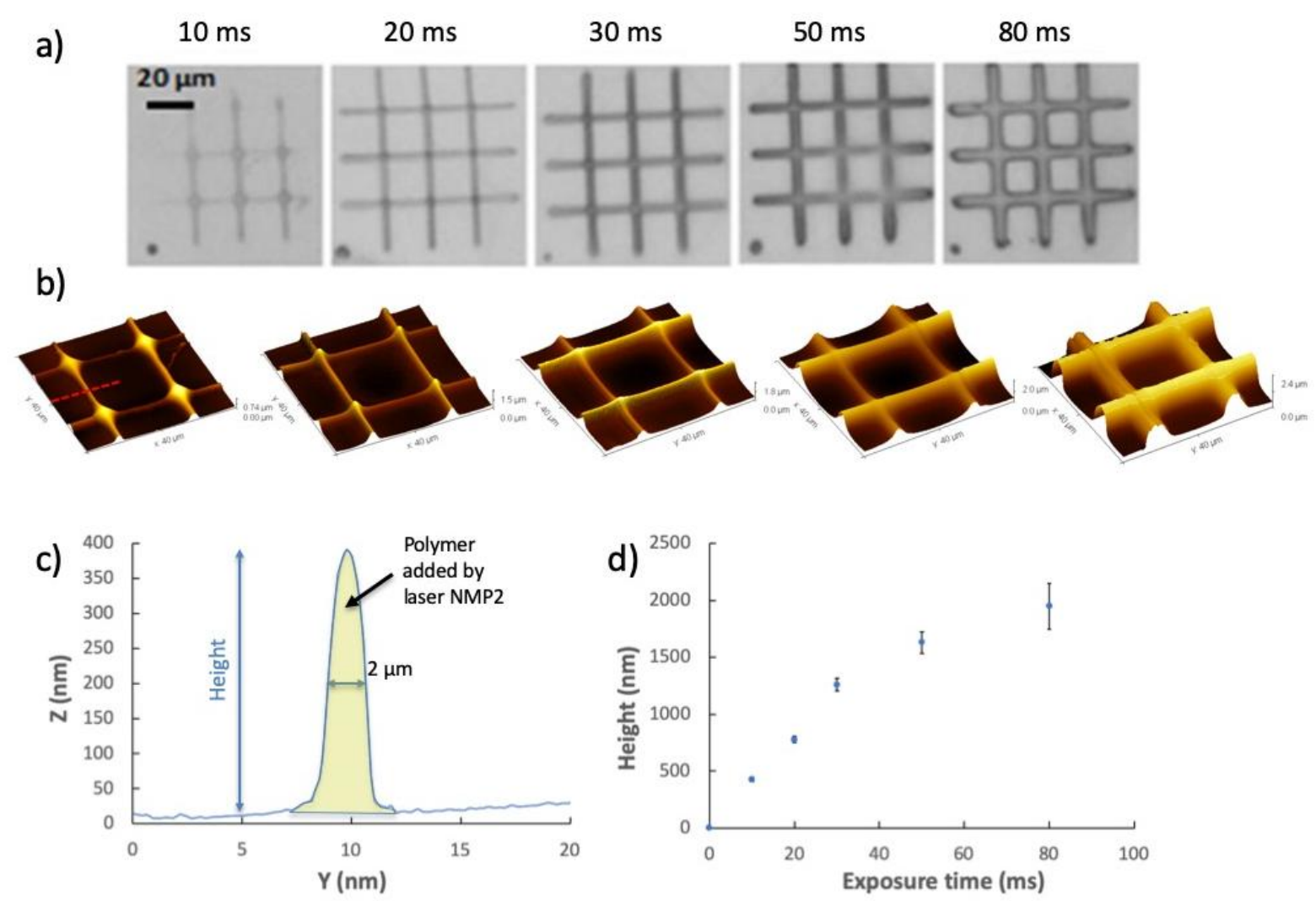

Figure 2: a) Optical microscopy images of the poly(TMPTA) microstructures written by NMP2 on an E605/AA film with a $375 \mathrm{~nm}$ laser $(3 \mu \mathrm{W})$. b) Same structures characterized by AFM. c) Representative profile of a line obtained by AFM (10 ms irradiation, along the red line). d) Evolution of the height of the structures with laser irradiation time $(3 \mu \mathrm{W})$. The height is measured from AFM profiles, between the first polymer surface and the top of the polymer structures.

Optical microscopy and AFM images (Figure 2.a and 2.b, respectively) show that this approach can be used to accurately produce microstructures on the surface of the sample. Control experiments have been carried out using the same monomers with commercial UV photoinitiator $(0.5 \mathrm{wt} \%$ of Irgacure 819 from Ciba). Using the same laser irradiation conditions, no polymer was formed at the surface. With the alkoxyamine, for the shortest writing times, the width of the structures is approximately $2 \mu \mathrm{m}$ (Figure 2.c), showing that microscale lateral resolution can be obtained. The evolution of the line heights $(\Delta \mathrm{h})$ versus exposure time is shown in Figure 2.d. $\Delta \mathrm{h}$ increases as a function of irradiation time, from $400 \mathrm{~nm}$ to $2000 \mathrm{~nm}$. For irradiation times under $50 \mathrm{~ms}$, the height linearly increases with 
irradiation time, which shows that good control of the microstructure height can be achieved by tuning a simple parameter such as the irradiation time.

For longer irradiation times, the growth slows down, indicating some limitations in the vertical propagation of the polymerization reaction. This can be explained by irreversible reactions such as inhibition by oxygen. This point will be further discussed below. However, the maximum height (2 $\mu \mathrm{m})$ is remarkable for a reaction initiated on the surface of the first film.

It is interesting to observe in Figure 2.b that for short irradiation times, the thickness in the crossing areas of the lines is double that of the single lines for short irradiation times. In contrast, for the longest irradiation times, the second laser pass did not increase the thickness. This result is in accordance with the preservation of the living character of the polymer for moderate irradiation times, which makes it possible to increase the polymerized thickness by an identical value during a second pass. This property opens the door towards the fabrication of 3D structures, if the irradiation conditions are well-chosen as shown below (see Fig. 4). In contrast, when the line is written with a dose corresponding to the saturation regime $(80 \mathrm{~ms})$, a second pass does not increase the thickness, which confirms the loss of reactivable species under such conditions.

We observed in previous studies that the irradiation power is an important parameter influencing the final result by conditioning the production kinetics of reactive species [27]. The power of the laser beam was therefore varied to investigate its influence on the spatial extent of the repolymerization. Lines were prepared with three laser powers: $0.550,0.340$ and $0.235 \mu \mathrm{W}$, corresponding to 2800,1750 and $1200 \mathrm{~mW} / \mathrm{cm}^{2}$, respectively (value estimated for a circular beam with a diameter of $5 \mu \mathrm{m}$ ). This power range is far below the power values chosen previously for the experiments in Fig 2.a because we are targeting here thin structures. Note that no structure was observed for powers below $0.235 \mu \mathrm{W}$, so this gave the minimum limit of the power that could be used for repolymerization. 


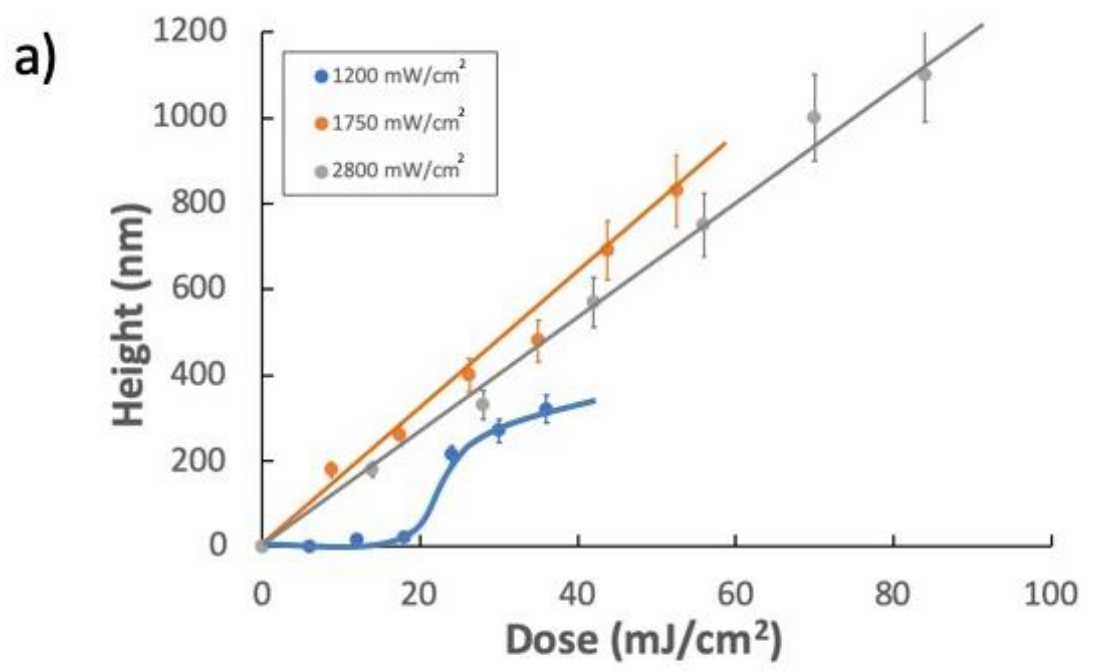

b)

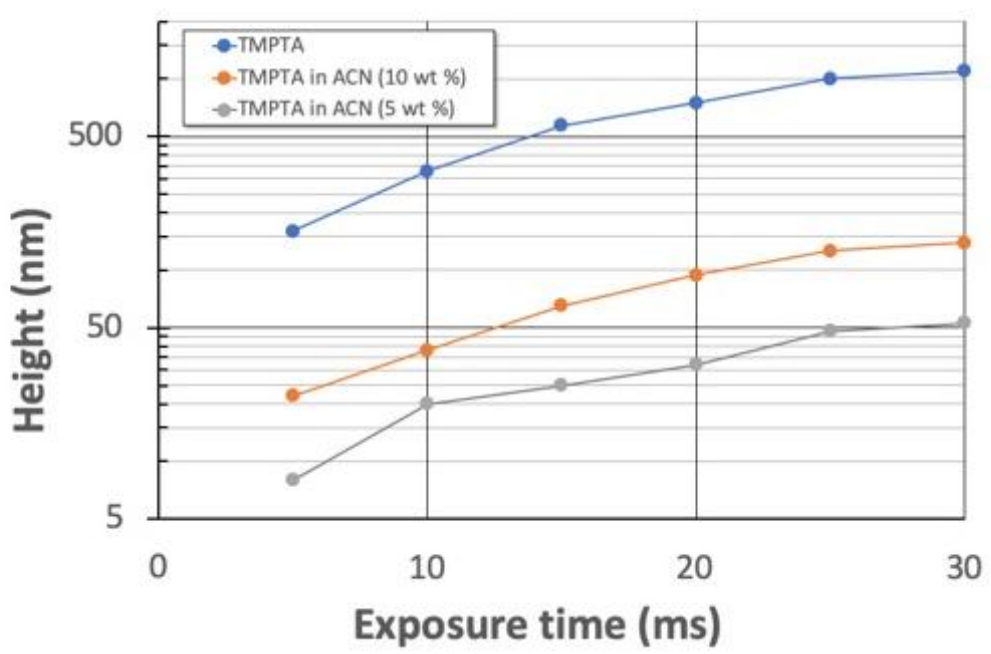

Figure 3. a) Effect of laser power on the height $(\Delta h)$ of laser-written structures with TMPTA as second monomer. Results are plotted here versus dose $\left(\mathrm{mJ} / \mathrm{cm}^{2}\right)$, defined as the power density $\left(\mathrm{mW} / \mathrm{cm}^{2}\right)$ multiplied by irradiation time. b) Effect of the dilution of the second monomer on the height $(\Delta \mathrm{h})$ of laser-written structures with an objective to obtain ultrathin layers (pure TMPTA and TMPTA diluted in acetonitrile with 10 and 5 wt. \%). Power density was chosen as $0.55 \mu \mathrm{W}$.

For a given power, the lines were written at different exposure times (from 5 to $30 \mathrm{~ms}$ ). The polymerization was carried out as before on an E605/AA film with TMPTA as the second monomer in all cases. The changes in the heights of polymer lines are shown in Figure 3.a as a function of dose so that the series can be compared with each other. 
In all cases, we observed, as expected, an increase in height with dose. For powers of 2800 and 1750 $\mathrm{mW} / \mathrm{cm}^{2}$, the increase in $\Delta \mathrm{h}$ was linear. with good control over the structure height.

For the lowest power $\left(1200 \mathrm{~mW} / \mathrm{cm}^{2}\right)$, an induction period was observed at low dose. Indeed, there is no polymerization for doses below $15 \mathrm{~mJ} / \mathrm{cm}^{2}$. The line heights could range from 200 to $1200 \mathrm{~nm}$ for doses greater than $20 \mathrm{~mJ} / \mathrm{cm}^{2}$. The heights were thus found slightly lower for the lowest writing power. This difference can be explained the influence of oxygen acting as an inhibitor of the free radical polymerization. Oxygen is indeed present in the acrylate resin before irradiation and its effect on the free radical polymerization kinetic is more important when the power is low.

The first results presented above show that it is possible to control the polymerized thickness but that it is difficult to obtain thicknesses below $150 \mathrm{~nm}$. Obtaining thin layers is important if a change in surface chemistry is targeted, without a change in dimensions. To obtain thinner structures, we proposed the use of a diluted solution of the monomer in an inert solvent (acetonitrile). The same laser conditions were then used (TMPTA as the second monomer on the E605/AA film polymerized for 50 $\mathrm{s}$ at $22 \mathrm{~mW} / \mathrm{cm}^{2}$ ). Figure 3.b shows the evolution of the height vs exposure time in the case of a polymerization reaction reactivated by pure TMPTA and TMPTA diluted to $10 \%$ and $5 \%$ by weight in acetonitrile. Solutions of TMPTA were used as pure monomer, as schematized in Figure 1. Under such conditions, the heights could be tuned from a few nanometers to a few micrometers. As expected, the more diluted monomer generates a lower $\Delta \mathrm{h}$.

It is thus possible to very finely control the polymerized thickness. For example, for a short exposure time ( $5 \mathrm{~ms}$ ), the heights are $200 \mathrm{~nm}$ for pure TMPTA, $35 \mathrm{~nm}$ for TMPTA diluted at $10 \%$ and $7 \mathrm{~nm}$ for TMPTA diluted at 5\%. This confirms that dilution of the monomer can be efficiently used to generate ultrathin layers through a combination of two phenomena: on the one hand, there is less monomer available at the reactivable surface for grafting reactions, and on the other hand, diluted conditions favor the recombination of radicals and thus limit the thickness.

The advantage of finely controlling the polymerized thickness is to be able to achieve, by this technique, either local chemical surface functionalization by conducting repolymerization with a monomer different from the first film, guaranteeing thicknesses of less than $5 \mathrm{~nm}$, or surface 
topography patterns with the same chemistry or a different chemistry between the polymer film and the microstructures.

Other examples of the high versatility of this approach are given in Figure 4. Figure 4.a shows the possibility of combining different chemistries. The two monomers chosen here are HEA and TMPTA, but this can be applied to any other acrylate monomer. These two monomers were chosen for their different hydrophilicities (contact angles with water are $70^{\circ}$ for TMPTA and $55^{\circ}$ for HEA).

Polymerization is carried out in two stages. First, a first monomer (TMPTA) is deposited on an E605/AA film polymerized under normal conditions. The laser is used to initiate a polymerization reaction. Parallel lines are thus made, and the sample is rinsed with ethanol and then dried.

Then, a second monomer (HEA) is deposited on the E605/AA film, and the laser is used to initiate another polymerization reaction. Lines are produced, perpendicular to those previously made. Finally, the sample is rinsed with ethanol. At the end, the sample consists of a grid with lines made from the 2 different polymers covalently grafted to a first polymer surface.
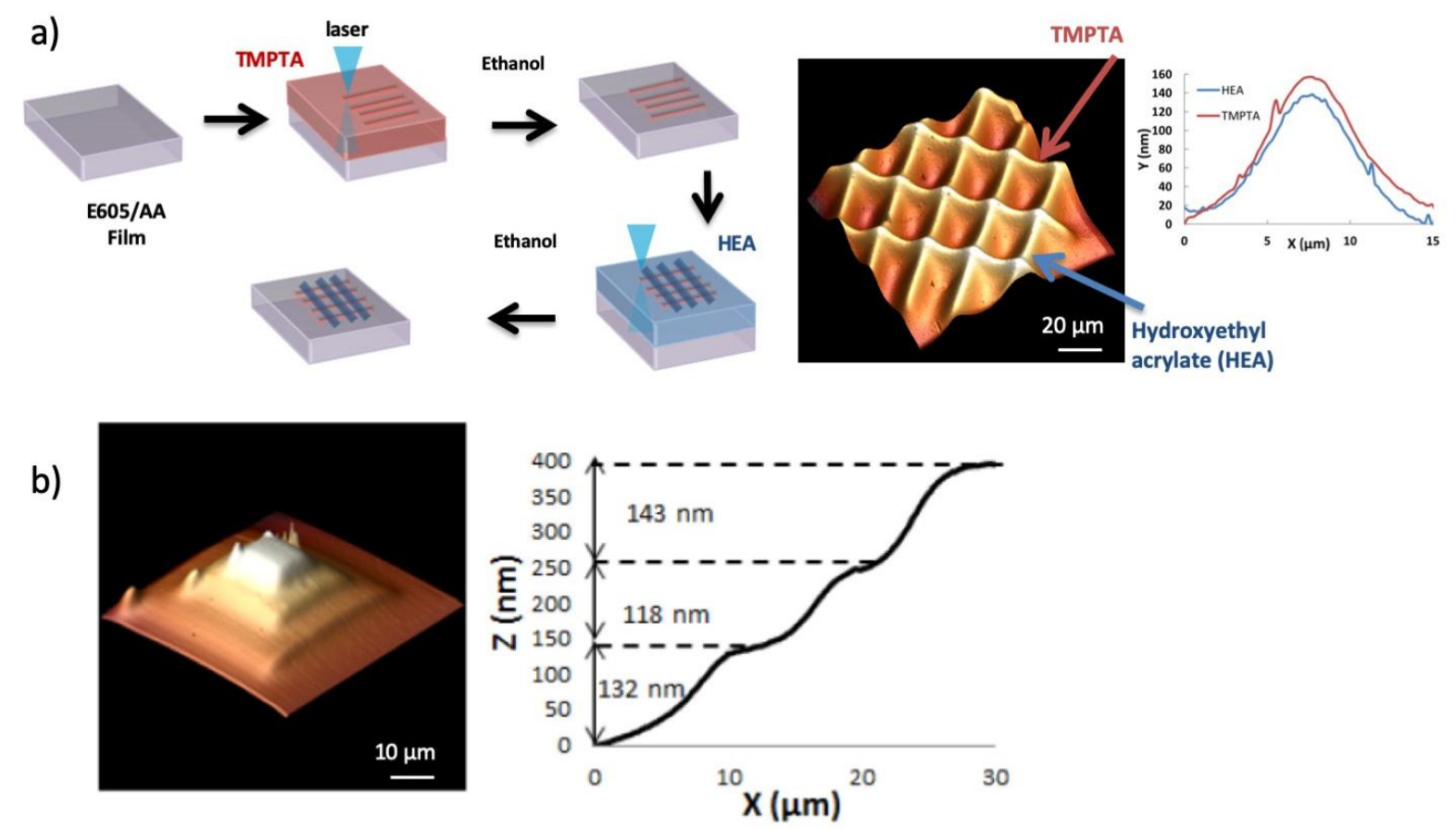

Figure 4. a) Schematic of the process for preparing multichemistry structures, and AFM analysis of a poly(HEA)/poly(TMPTA) grid, with analysis of the structure height by AFM for both chemistries. b) 3D object made by successive writing imaged by AFM (right : section profile). 
At a given power of $2.9 \mu \mathrm{W}$ and an exposure time of $10 \mathrm{~ms}$, the heights of the lines formed with poly(HEA) are the same as those formed with poly(TMPTA), as shown in Figure 4.a. This can of course be applied to more monomers to generate multichemistry microstructures.

The possibility of tuning the repolymerized thickness and sequentially growing several layers enables the generation of 3D structures (Figure 4.b). In this example, on an E605/AA film irradiated under usual conditions ( $50 \mathrm{~s}$ to $22 \mathrm{~mW} / \mathrm{cm}^{2}$ ), square structures were sequentially written. The dimensions of the squares at each level are 50, 30 and $15 \mu \mathrm{m}$. For each level, the laser exposure time was adjusted to generate an identical height for each stage. There is no washing step between the fabrication of each layer but only when the 3 layers were prepared. In a first step, the $50 \mu \mathrm{m}$ square was written at the surface of the polymer layer prepared as in Figure 1. The square is written by as lines with overlap. Then the $30 \mu \mathrm{m}$ was written in the middle of the first square and finally, the last square was written. Figure 4.b shows that for a fixed power and variable exposure times of $1 \mathrm{~ms}, 4 \mathrm{~ms}$ and $20 \mathrm{~ms}$, the polymerized thicknesses are of the same order of magnitude. Indeed, the heights are $132 \mathrm{~nm}, 118 \mathrm{~nm}$ and $143 \mathrm{~nm}$. This example demonstrates the potential of this technique to generate 3D shapes but shows that a significant loss of reactivity is observed for each level. As already mentioned, this behavior was attributed to side reactions of radical species with oxygen. The objective of the last part of this paper is to illustrate the role of oxygen in this context.

Oxygen is known to be a strong inhibitor of free radical polymerization (Figure 5.a). For bulk free radical polymerization, oxygen is thus usually minimized in the medium to optimize the polymerization rate and final conversion. Many strategies have been developed to maintain the performance of photopolymerization under the presence of oxygen, including in the context of controlled/living radical polymerization.[29] However, when spatial control of polymerization at the micro- and even nanoscale is desired, the presence of an inhibitor is needed. [30,31,32] Here, we would like to discuss the role of oxygen and answer the question of whether the z-containment of the polymerization is essentially ensured by the NMP2 mechanism or whether oxygen inhibition plays an important role. We already mentioned that some results of the repolymerization by NMP2 on the 
surface may be explained by reactivity of the system to oxygen (results at low power in Figure 3.a, low thickness of the polymerized layer in the sequential fabrication process in Figure 4.b).

The repolymerization was begun by sweeping the sample with a flow of $\mathrm{N}_{2}$, which eliminated oxygen from the atmosphere around the polymer film and in the monomer. Other conditions were kept identical to the previous ones, except that for practical reasons, the sample was irradiated through the substrate and E605/AA film in this configuration. Preliminary tests were carried out to show that 10 min under nitrogen flow before polymerization was sufficient to remove the oxygen in the TMPTA monomer.
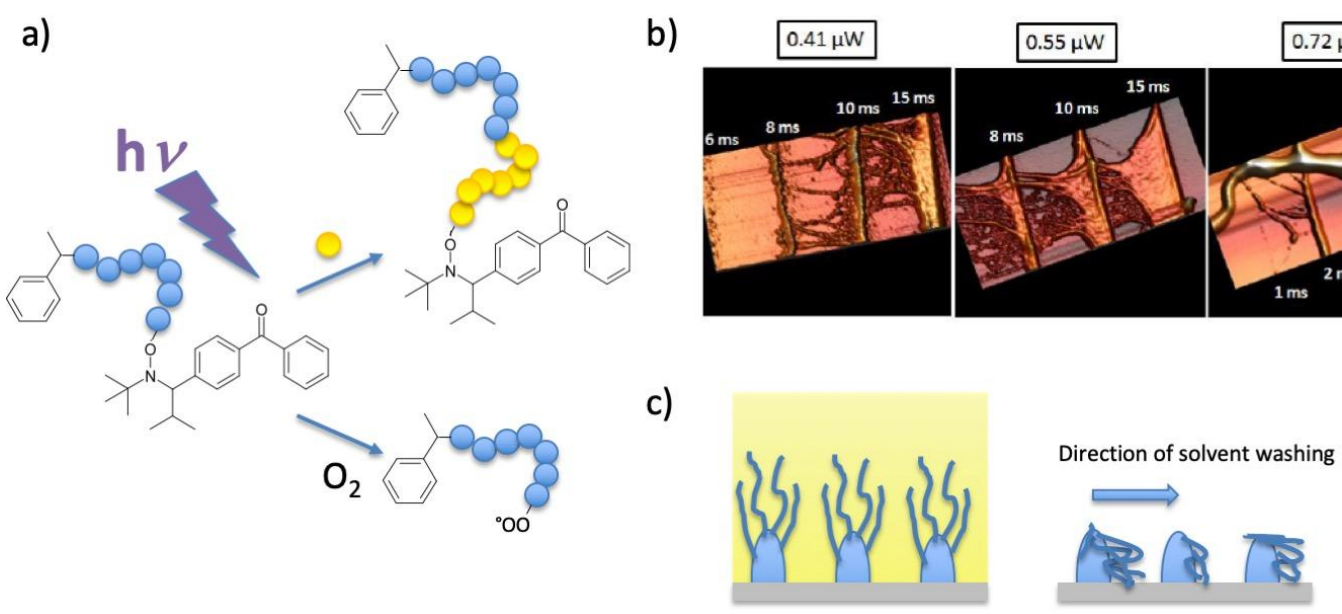

Figure 5. a) Illustration of the competition between repolymerization by NMP2 and quenching of radicals by $\mathrm{O}_{2}$, leading to inactive radicals. b) AFM images of the lines prepared with different laser powers under nitrogen after development with ethanol. c) Schematic of the structure of the polymer lines after laser writing, accounting for the shape of the lines after development.

Lines were prepared at different exposure times ranging from 1 to $15 \mathrm{~ms}$ and variable powers from 0.31 to $0.92 \mu \mathrm{W}$ (measured at the output of the $\mathrm{x} 100$ microscope objective). After polymerization by the laser beam, the sample was rinsed with ethanol as before. We would first like to emphasize that under air, equivalent thicknesses were obtained for exposure times between 10 and $80 \mathrm{~ms}$ at $3 \mu \mathrm{W}$. We note that there are no lines formed for an exposure time of $1 \mathrm{~ms}$, regardless of the power used, and that there is no polymerization at $0.31 \mu \mathrm{W}$. Therefore, experiments conducted under $\mathrm{N}_{2}$ show that the system is approximately 50 times more efficient under $\mathrm{N}_{2}$ than under air. The microscopy images 
obtained after rinsing reveal different structures than the smooth lines obtained under air. Indeed, the lines show significant roughness, which is accentuated when the laser exposure time is high. The structures were observed by AFM, and the most relevant images are shown in Figure 5.b.

At a power of $0.41 \mu \mathrm{W}$, the lines do not have a regular appearance, and polymer "filaments" are observed between them. The amount of these filaments appears to increase with exposure time. Note that at $0.72 \mu \mathrm{W}$, the fiber structure observed perpendicular to the written lines may result from the collapsing of bigger filament structures.

These structures were systematically obtained for all experiments carried out in the absence of oxygen. The filaments seem to have fallen in a given direction, which was ascribed to the sample rinsing direction during development, as schematically depicted in Figure 5.c. This was also supported by optical microscopy observations prior to development, showing well defined lines before development, which means that the filament structures were perpendicular to the observation direction (perpendicular to the substrate).

These filamentary structures can be explained by the absence of the inhibitor in the medium, leading to uncontrolled spatial propagation of free radical polymerization. This means that the spatial control of the polymerization reaction is not achieved only by the recombination of radicals, as expected in the NMP2 molecular pathway, but that oxygen is also needed to confine the reaction under laser irradiation conditions.

This unique behavior may be explained on the basis of the very specific regime of laser irradiation. The high-power density generated implies a high concentration of photoinduced reactive species, which does not permit sufficient polymer/nitroxide recombination reactions to occur. In the end, this series of experiments shows the ambivalent role of oxygen: On the one hand, oxygen has a negative role in the polymerization process by inducing irreversible termination reactions, which limits the possible extension of the polymerization. On the other hand, oxygen also plays a positive role in the context of microstructuring by limiting the spatial extent of the free radical polymerization. It is therefore preferential to work in the presence of oxygen in this laser writing configuration.

\section{Conclusion}


In conclusion, NMP2 by direct laser writing has made it possible to produce polymer microstructures with variable architectures. The laser beam was used in different configurations. It has been shown that laser irradiation can be used to fabricate microstructures on a polymer film or to directly fabricate laser structures that can be functionalized by NMP2.

Conditions allowing control of the polymerized thickness could be defined. Although the power densities are much higher than in the case of UV lamp irradiation, thicknesses as thin as a few tens of nanometers can be reproducibly obtained using monomers diluted in a solvent and a low power. Spatial control of polymerization is also directly related to the inhibitory effect of oxygen. Although the presence of oxygen in the polymerization medium is a negative factor for the sensitivity of the system (the system is 50 times less reactive under air than under nitrogen), its presence is essential to obtain well-defined structures.

These conditions have made it possible to fabricate complex multilayer microstructures with fine zcontrol. It has also been shown that it is possible to combine different chemistries in the same structure. These results can lead to different applications in the field of biomaterials or sensors.

\section{Acknowledgements}

This work was supported by funding from Agence Nationale pour la Recherche (Project IMPACT, ANR2011BS08016).

\section{Table Of Contents (TOC) graphic}

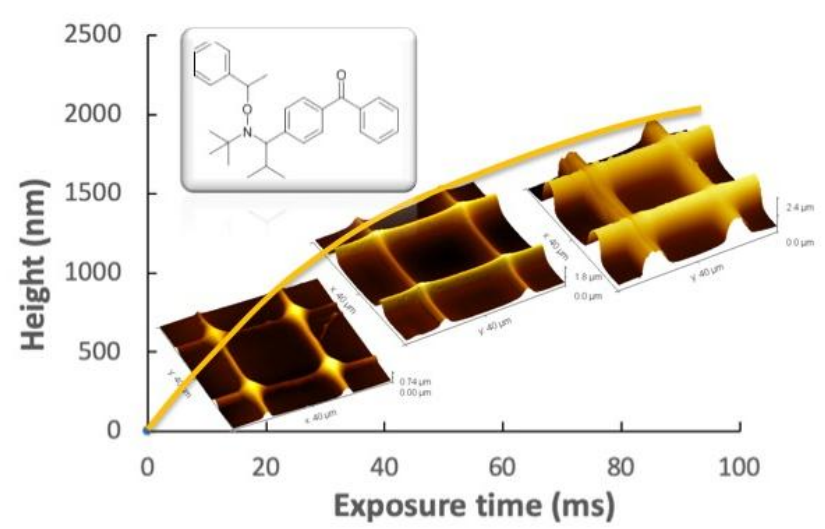




\section{References}

${ }^{1}$ Menon, R.; Patel, A.; Gil, D.; Smith, H. I., Maskless Lithography. Materials Today 2005, 8 (2), 2633.

${ }^{2}$ Yu, S. Y.; Schrodj, G.; Mougin, K.; Dentzer, J.; Malval, J. P.; Zan, H. W.; Soppera, O.; Spangenberg, A., Direct Laser Writing of Crystallized TiO2 and TiO2/Carbon Microstructures with Tunable Conductive Properties. Advanced Materials 2018, 30 (51), 8.

${ }^{3}$ Yeh, C. C.; Zan, H. W.; Soppera, O., Solution-Based Micro- and Nanoscale Metal Oxide Structures Formed by Direct Patterning for Electro-Optical Applications. Advanced Materials 2018, 30 (50), 24.

${ }^{4}$ Baron, M.; Morris, J. C.; Telitel, S.; Clement, J. L.; Lalevee, J.; Morlet-Savary, F.; Spangenberg, A.; Malval, J. P.; Soppera, O.; Gigmes, D.; Guillaneuf, Y., Light-Sensitive Alkoxyamines as Versatile Spatially- and Temporally-Controlled Precursors of Alkyl Radicals and Nitroxides. J Am Chem Soc 2018, 140 (9), 3339-3344.

${ }^{5}$ Lin, H.-C.; Stehlin, F.; Soppera, O.; Zan, H.-W.; Li, C.-H.; Wieder, F.; Ponche, A.; Berling, D.; Yeh, B.-H.; Wang, K.-H., Deep Ultraviolet Laser Direct Write for Patterning Sol-gel InGaZnO Semiconducting Micro/Nanowires and Improving Field-Effect Mobility. Scientific Reports 2015, 5, 10410.

${ }^{6}$ Stehlin, F.; Wieder, F.; Spangenberg, A.; Le Meins, J.-M.; Soppera, O., Room-Temperature Preparation of Metal-Oxide Nanostructures by DUV Lithography from Metal-oxo Clusters. Journal of Materials Chemistry C 2014, 2, 277-285.

${ }^{7}$ Malinauskas, M.; Žukauskas, A.; Hasegawa, S.; Hayasaki, Y.; Mizeikis, V.; Buividas, R.; Juodkazis, S., Ultrafast Laser Processing of Materials: From Science to Industry. Light: Science \& Applications 2016, 5 (8), e16133-e16133.

${ }^{8}$ Chang, P.-Y.; Bruntz, A.; Vidal, L.; Vetter, P.-A.; Roudot, P.; Bua, L.; Ortiz, J.; Zan, H.-W.; Soppera, O., Laser Polymer Tattooing: A Versatile Method for Permanent Marking on Polymer Surfaces. Macromol Mater Eng 2019, 1900402.

${ }^{9}$ Aekbote, B. L.; Jacak, J.; Schütz, G. J.; Csányi, E.; Szegletes, Z.; Ormos, P.; Kelemen, L., Aminosilane-Based Functionalization of Two-Photon Polymerized 3D SU-8 Microstructures. European Polymer Journal 2012, 48 (10), 1745-1754.

${ }^{10}$ Aekbote, B. L.; Fekete, T.; Jacak, J.; Vizsnyiczai, G.; Ormos, P.; Kelemen, L., Surface-Modified Complex SU-8 Microstructures for Indirect Optical Manipulation of Single Cells. Biomed Opt Express 2015, 7 (1), 45-56.

${ }^{11}$ Farrer, R. A.; LaFratta, C. N.; Li, L.; Praino, J.; Naughton, M. J.; Saleh, B. E. A.; Teich, M. C.; Fourkas, J. T., Selective Functionalization of 3-D Polymer Microstructures. Journal of the American Chemical Society 2006, 128 (6), 1796-1797.

${ }^{12}$ Quick, A. S.; Fischer, J.; Richter, B.; Pauloehrl, T.; Trouillet, V.; Wegener, M.; Barner-Kowollik, C., Preparation of Reactive Three-Dimensional Microstructures via Direct Laser Writing and Thiolene Chemistry. Macromolecular Rapid Communications 2013, 34 (4), 335-340.

${ }^{13}$ Wang, X.; Cai, X.; Guo, Q.; Zhang, T.; Kobe, B.; Yang, J., i3DP, A Robust 3D Printing Approach Enabling Genetic Post-Printing Surface Modification. Chemical Communications 2013, 49 (86), 10064-10066. 
${ }^{14}$ Yee, D. W.; Schulz, M. D.; Grubbs, R. H.; Greer, J. R., Functionalized 3D Architected Materials via Thiol-Michael Addition and Two-Photon Lithography. Advanced Materials 2017, 29 (16), 1605293.

${ }^{15}$ Telitel, S.; Dumur, F.; Telitel, S.; Soppera, O.; Lepeltier, M.; Guillaneuf, Y.; Poly, J.; MorletSavary, F.; Fioux, P.; Fouassier, J. P.; Gigmes, D.; Lalevee, J., Photoredox Catalysis Using a New Iridium Complex as an Efficient Toolbox for Radical, Cationic and Controlled Polymerizations under Soft Blue to Green Lights. Polymer Chemistry 2015, 6 (4), 613-624.

${ }^{16}$ Richter, B.; Pauloehrl, T.; Kaschke, J.; Fichtner, D.; Fischer, J.; Greiner, A. M.; Wedlich, D.; Wegener, M.; Delaittre, G.; Barner-Kowollik, C.; Bastmeyer, M., Three-Dimensional Microscaffolds Exhibiting Spatially Resolved Surface Chemistry. Advanced Materials 2013, 25 (42), 6117-6122.

${ }^{17}$ Quick, A. S.; de los Santos Pereira, A.; Bruns, M.; Bückmann, T.; Rodriguez-Emmenegger, C.; Wegener, M.; Barner-Kowollik, C., Rapid Thiol-Yne-Mediated Fabrication and Dual Postfunctionalization of Micro-Resolved 3D Mesostructures. Advanced Functional Materials 2015, 25 (24), 3735-3744.

${ }^{18}$ Claus, T. K.; Richter, B.; Hahn, V.; Welle, A.; Kayser, S.; Wegener, M.; Bastmeyer, M.; Delaittre, G.; Barner-Kowollik, C., Simultaneous Dual Encoding of Three-Dimensional Structures by LightInduced Modular Ligation. Angewandte Chemie International Edition 2016, 55 (11), 3817-3822.

${ }^{19}$ Chen, M.; Gu, Y.; Singh, A.; Zhong, M.; Jordan, A. M.; Biswas, S.; Korley, L. T. J.; Balazs, A. C.; Johnson, J. A., Living Additive Manufacturing: Transformation of Parent Gels into Diversely Functionalized Daughter Gels Made Possible by Visible Light Photoredox Catalysis. ACS Central Science 2017, 3 (2), 124-134.

${ }^{20}$ Chen, M., Zhong, M. \& Johnson, J. A. Light-Controlled Radical Polymerization: Mechanisms, Methods, and Applications. Chem. Rev. 116, 10167-10211 (2016).

${ }^{21}$ Pan, X., Tasdelen, M.A., Laun, J., Junkers, T., Yagci, Y., Matyjaszewski, K. Photomediated Controlled Radical Polymerization. Prog. Polym. Sci. 62, 73-125 (2016).

${ }^{22}$ Dadashi-Silab, S., Doran, S. \& Yagci, Y. Photoinduced Electron Transfer Reactions for Macromolecular Syntheses. Chem. Rev. 116, 10212-10275 (2016).

${ }^{23}$ Corrigan, N., Shanmugam, S., Xu, J. \& Boyer, C. Photocatalysis in Organic and Polymer Synthesis. Chem. Soc. Rev. 45, 6165-6212 (2016).

${ }^{24}$ Zhang, Z., Corrigan, N., Bagheri, A., Jin, J., Boyer, C., A Versatile 3D and 4D Printing System through Photocontrolled RAFT Polymerization, Angewandte Chemie International Edition 2019, 131 (50), 18122-18131.

${ }^{25}$ Li, M., Fromel, M., Ranaweera, D., Rocha, S., Boyer, C., Perster, C.W., SI-PET-RAFT: SurfaceInitiated Photoinduced Electron Transfer-Reversible Addition-Fragmentation Chain Transfer Polymerization, ACS Macro Letters 2019, 8 (4), 374-380

${ }^{26}$ Jung, K., Corrigan, N., Ciftci, M., Xu, J., Seo, S.E., Hawker, C.J., Boyer, C., Designing with Light: Advanced 2D, 3D, and 4D Materials, Advanced Materials 2019, 1903850.

${ }^{27}$ Telitel, S.; Telitel, S.; Bosson, J.; Lalevee, J.; Clement, J. L.; Godfroy, M.; Fillaut, J. L.; AkdasKilig, H.; Guillaneuf, Y.; Gigmes, D.; Soppera, O., UV-Induced Micropatterning of Complex 
Functional Surfaces by Photopolymerization Controlled by Alkoxyamines. Langmuir 2015, 31 (36), 10026-10036.

${ }^{28}$ Telitel, S.; Telitel, S.; Bosson, J.; Spangenberg, A.; Lalevee, J.; Morlet-Savary, F.; Clement, J. L.; Guillaneuf, Y.; Gigmes, D.; Soppera, O., Nitroxide Mediated Photopolymerization: A Versatile Tool for the Fabrication of Complex Multilayer Polyfunctional Copolymer Nanostructures. Advanced Materials Interfaces 2014, 1, 1400067.

${ }^{29}$ Yeow, J., Chapman, R., Gormley, A.J., Boyer, C. Up in the Air: Oxygen Tolerance in Controlled/Living Radical Polymerization, Chem Soc Rev. 2018, 47, 4357-4387.

${ }^{30}$ Croutxe-Barghorn, C.; Soppera, O.; Simonin, L.; Lougnot, D. J., On the Unexpected Role of Oxygen in the Generation of Microlens Arrays with Self-Developing Photopolymers. Adv Mater Opt Electr 2000, 10 (1), 25-38.

${ }^{31}$ Soppera, O.; Jradi, S.; Lougnot, D. J., Photopolymerization with Microscale Resolution: Influence of the Physico-Chemical and Photonic Parameters. J Polym Sci Pol Chem 2008, 46 (11), 3783-3794.

${ }^{32}$ Deeb, C.; Ecoffet, C.; Bachelot, R.; Plain, J.; Bouhelier, A.; Soppera, O., Plasmon-Based FreeRadical Photopolymerization: Effect of Diffusion on Nanolithography Processes. J Am Chem Soc 2011, 133 (27), 10535-10542. 